\title{
DISCRETE SYMMETRY, TORAL SYMMETRY AND THE EULER CHARACTERISTIC OF MANIFOLDS
}

\author{
STEFAN PAPADIMA
}

(Communicated by Haynes R. Miller)

\begin{abstract}
We consider a closed manifold $M$ and establish various connections between its Euler characteristic and certain numerical invariants defined so as to measure the abundance of free discrete (respectively rationally free toral) group actions supported by $M$.
\end{abstract}

1. Introduction. Let $M$ be closed connected differentiable manifold. The toral rank of $M$ is defined (following W.-Y. Hsiang, see [7, p. 131]) by $\operatorname{trk}(M)=\operatorname{maximum}$ $r$ for which there exists a rationally free action (i.e. with only finite isotropy groups) of the torus $T^{r}$ on $M$. This invariant was intensively studied in the theory of compact Lie transformation groups, see e.g. $[\mathbf{1}, \mathbf{2}, \mathbf{6}]$ (to quote only work directly related to the present note). We shall also consider (following $[\mathbf{1 0}$, p. 639]) the free symmetry index of $M$, denoted by $f(M)$, defined by $f(M)=\infty$ if there exists a free action of an infinite discrete group on $M$ (we only demand that all isotropy groups are trivial - the action is not supposed to be properly discontinuous!), and otherwise by $f(M)=$ maximum of the cardinalities of the finite groups which can act freely on $M$.

The main result of this note characterizes the finiteness property for $f(M)$ and establishes the relationship between this property and the vanishing of $\operatorname{trk}(M)$. 0 .

THEOREM. $f(M)<\infty$ if and only if $\chi(M) \neq 0$. If $f(M)<\infty$ then $\operatorname{trk}(M)=$

This result was stated (without proof) in [10, Remarks 4.2]. Its proof occupies the next section. In $\S 3$ we shall make several comments on it and present a few relevant examples.

2. Proof of the Theorem. Suppose that the Euler characteristic is nonzero. We claim that it suffices to show that no infinite discrete group can act freely on $M$. Once we know this, it immediately follows that $f(M) \leq|\chi(M)|$, in particular $f(M)<\infty$ (since, as is well known, a free action of a finite group $G$ on $M$ gives rise to a finite covering $M \rightarrow M / G$ for which one has $\chi(M)=\operatorname{ord}(G) \cdot \chi(M / G))$.

Suppose then that the discrete group $G$ acts freely on $M$. We are going to show that then necessarily ord $(G)<\infty$. As a first step towards proving this, let us notice that there exists an integer $m$ with the property that $\operatorname{ord}(g) \leq m$, for any $g \in G$. Indeed, if $\chi(M) \neq 0$ then given $g \in G$, the classical Lefschetz number argument of Fuller [5] shows that $g^{n}$ must have a fixed point, for some $0<n \leq m$, where $m$ depends only on $M$, hence $g^{n}=\mathrm{id}$ and $\operatorname{ord}(g) \leq m$.

Received by the editors February 27, 1987.

1980 Mathematics Subject Classification (1985 Revision). Primary 57S17, 57S15.

Key words and phrases. Euler characteristic, free (rationally free) action. 
Observe next that sending $g \in G$ into $H^{*}\left(g^{-1} ; \mathbf{F}\right) \in G L\left(H^{*}(M ; \mathbf{F})\right)$ embeds the group $G$ into the general linear group of the finite-dimensional $\mathbf{F}$-vector space $H^{*}(M ; \mathbf{F})$, for any characteristic zero field coefficients $\mathbf{F}$ (using again Lefschetz numbers, $\chi(M) \neq 0$ and the freeness of the action of $G$ ).

The next lemma helps to conclude that $\operatorname{ord}(G)$ is finite.

LEMMA. Let $\mathbf{F}$ be a characteristic zero algebraically closed field and let $G \subset$ $G L(n ; \mathbf{F})$ be a subgroup. If there exists an integer $m$ such that $\operatorname{ord}(g) \leq m$, for any $g \in G$, then $G$ is finite.

PROOF. The proof uses (elementary) linear algebraic groups theory [8]. We may clearly suppose that $G$ is a (Zariski) closed subgroup. Moreover, it will be enough to show that if in addition $G$ is connected then $G$ must be trivial. Since all the elements of $G$ have finite order, we infer that $G$ consists only of semisimple elements (remember that char $\mathbf{F}=0$ !). But then $G$ must be an (algebraic) torus $\left(\mathbf{F}^{*}\right)^{r}$, see $\left[\mathbf{8}\right.$, p. 137]. The fact that $\mathbf{F}^{*}$ contains elements of any given order forces then $r=0$ and consequently $G=\{1\}$.

Passing to the other implication of the first statement of our theorem, let us assume. $\chi(M)=0$. We are going to produce a free action of $\mathbf{Z}$ on $M$ and infer that $f(M)=\infty$. Though we are looking for a discrete dynamical system, we shall construct it by considering flows on $M$, namely by using properties of hyperbolic flows, see [11]. This is the only place where differentiability assumptions play a significant part.

By the Poincaré-Hopf theorem there exists a nowhere zero vector field $X$ on $M$. It gives rise to a flow $g_{t}, t \in \mathbf{R}$, with no fixed points. In general, given any vector field $X$ on $M$, with associated flow $g_{t}: \mathbf{R} \times M \rightarrow M$, consider the closed orbits $\mathbf{R} \cdot x$ $\left(x \in M\right.$ ), defined by $\mathbf{R}_{x}=\mathbf{Z} \cdot t$ (for some period $t>0$ ). Define the set of periods of $X$ by $P_{X}=\left\{t>0 \mid\right.$ there exists $x \in M$ such that $\left.\mathbf{R}_{x}=\mathbf{Z} \cdot t\right\}$. One knows that generically $P_{X} \cap[0, c]$ is finite, for any $c>0$, see $[11$, p. 801]. We may thus suppose that our everywhere nonzero vector field $X$ also satisfies this generic property. Pick then $s \in \mathbf{R}, s \neq 0$ and not of the form $s=q \cdot t$, with $q \in \mathbf{Q}$ and $t \in P_{X}$. We claim that the flow $g_{t}$ restricted to $\mathbf{Z} \cdot s$ gives the desired free action of $\mathbf{Z}$ on $M$. Indeed, if $g_{m s}(x)=x$, for some $m \in \mathbf{Z}$ and $x \in M$, it follows that $\mathbf{R} \cdot x$ is a closed orbit and $m s=n t$, for some $n \in \mathbf{Z}$ and $t \in P_{X}$, which forces $m=0$.

Finally, we quickly prove the second assertion of the Theorem. Assume $f(M)<$ $\infty$ and let a torus $T$ act on $M$. We show that $T$ must have a fixed point on $M$ (and consequently $\operatorname{trk}(M)=0$ ). Pick a generator $z \in T$ (that is the group generated by $z$ is dense in $T$ ) and restrict the given action of $T$ to the (infinite cyclic) subgroup generated by $z$. Our assumption on $f(M)$ implies that $z^{m}$ has a fixed point, for some $m \in \mathbf{Z}, m \neq 0$. But $z^{m}$ is also a generator, hence $T$ must leave that point fixed.

\section{Examples.}

EXAMPLE 1. We might have restricted our attention to free actions of finite groups on $M$, defining $f f(M)=\max \{\operatorname{ord}(G) \mid G$ is finite and acts freely on $M\}$. Our theorem essentially says then that, if $\chi(M) \neq 0$, this makes no difference: $f f(M)=f(M)$, (and this common value is finite). On the other hand, there are examples of closed connected manifolds $M$ with zero Euler characteristic which do not even possess finite effective groups of homeomorphisms of arbitrarily high order, 
hence with $f f(M)<\infty$, see $[3,4]$. In all these (in fact aspherical) examples the fundamental group plays a key role. More recently, several classes of 1-connected examples with $\chi(M)=0$ have appeared (see $[9,2]$ ), which do admit free $\mathbf{Z}_{p^{-}}$ actions for almost all primes $p$, in particular with $f f(M)=\infty$ (the connected sums of manifolds of the form $M=S^{m} \times S^{n}$, which $m, n>1$ and $m+n=$ odd, are among them). This raises the question: is it true that $f f(M)=\infty$, for a 1-connected closed manifold $M$ with $\chi(M)=0$ ?

EXAMPLE 2. Going back to arbitrary closed connected manifolds $M$, recall from the proof of our theorem that if $\chi(M) \neq 0$ then $f(M) \leq|\chi(M)|$. It might happen that this upper bound is sharp, as it is the case for $M=G / T$ ( $G$ a compact connected Lie group, $T$ a maximal torus), where $f(M)=|\chi(M)|=$ order of the Weyl group (see [10]). However, this is a very crude estimate in general, as $M=\mathbf{P}^{2 n} \mathbf{C}$ shows: here any self-map has a fixed point, hence $f(M)=1$, whereas $\chi(M)=2 n+1$.

EXAMPLE 3. It is well known that on a finite complex with $\chi(M) \neq 0$ any torus action has a fixed point. Our aim here is to emphasize the connection with the finiteness of $f(M)$. The converse is not true in general, as shown by the following example of S. Halperin [6], who constructed a closed oriented manifold $M$ with $\operatorname{dim} M=57$ (hence $\chi(M)=0$ ) and $\operatorname{trk}(M)=0$. On the other hand, for homogeneous spaces $M=G / K$ (where $G$ is a compact connected Lie group and $K$ is a closed subgroup), one knows [1] that $\operatorname{trk}(M)=\operatorname{rk} G-\operatorname{rk} K$, hence $\operatorname{trk}(M)=0$ implies that $\chi(M) \neq 0$.

\section{BIBLIOGRAPHY}

1. C. Allday and S. Halperin, Lie group actions on spaces of finite rank, Quart. J. Math. Oxford Ser. 29 (1978), 63-76.

2. M. Aubry and J.-M. Lemaire, Sommes connexes fibrées en cercles, Bull. Soc. Math. France 113 (1985), 459-462.

3. A. Borel, On periodic maps on certain $K(\pi, 1)$, Collected Papers, vol. III, Springer-Verlag, 1983, pp. 57-60.

4. P. E. Conner and F. Raymond, Manifolds with few periodic homeomorphisms, Proc. Second Conf. on Compact Transformation Groups, Part II, Lecture Notes in Math., vol. 299, Springer-Verlag, Berlin and New York, 1972, pp. 1-75.

5. F. B. Fuller, The existence of periodic points, Ann. of Math. (2) 57 (1953), 229-230.

6. S. Halperin, Rational homotopy and torus actions, Aspects of Topology, Mem. H. Dowker, London Math. Soc. Lecture Note Ser. 93, 1985, pp. 293-306.

7. W.-Y. Hsiang, Cohomology theory of topological transformation groups, Springer-Verlag, 1975.

8. J. E. Humphreys, Linear algebraic groups, Springer-Verlag, 1975.

9. P. Löffler and M. Raussen, Symmetrien von Mannigfaltigkeiten und Rationale Homotopietheorie, Math. Ann. 271 (1985), 549-576.

10. S. Papadima, Rigidity properties of compact Lie groups modulo maximal tori, Math. Ann. 275 (1986), 637-652.

11. S. Smale, Differentiable dynamical systems, Bull. Amer. Math. Soc. 73 (1967), 747-817.

Department of Mathematics, INCREST, Bdul PaCil 220, 79622 Bucharest, ROMANIA 